\title{
Aromatic stacking interactions govern catalysis in aryl-alcohol oxidase
}

Patricia Ferreira ${ }^{1}$, Aitor Hernández-Ortega ${ }^{2 *}$, Fátima Lucas ${ }^{3}$, Juan Carro ${ }^{2}$, Beatriz Herguedas $^{1 \S}$, Kenneth W. Borrelli ${ }^{3,4 \#}$, Victor Guallar ${ }^{3,4}$, Angel T. Martínez $^{2}$ and Milagros Medina ${ }^{1}$

${ }^{1}$ Departamento de Bioquímica y Biología Molecular y Celular, Facultad de Ciencias, Universidad de Zaragoza, and Instituto de Biocomputación y Física de Sistemas Complejos (BIFI), Pedro Cerbuna, 12, E-50009 Zaragoza, Spain

${ }^{2}$ Centro de Investigaciones Biológicas, CSIC, Ramiro de Maeztu 9, E-28040 Madrid, Spain

${ }^{3}$ Joint BSC-CRG-IRB Research Program in Computational Biology, Barcelona Supercomputing Center, Jordi Girona 29, E-08034 Barcelona, Spain

${ }^{4}$ Institució Catalana de Recerca i Estudis Avançats (ICREA), Passeig Lluís Companys 23, E-08010

Barcelona, Spain

Present addresses

* MIB, University of Manchester, Manchester, UK

${ }^{\S}$ MRC Laboratory of Molecular Biology, Cambridge, UK

Short title

Substrate stacking interactions in aryl-alcohol oxidase

\section{Keywords}

Aryl-alcohol oxidase, GMC oxidoreductases, aromatic stacking, catalytic mechanism, ternary complex, ping-pong, charge-transfer complexes, kinetic isotope effect, steady-state kinetics, presteady-state kinetics

\section{Correspondence}

Patricia Ferreira, Departamento de Bioquímica y Biología Molecular y Celular, Facultad de Ciencias, Universidad de Zaragoza, Pedro Cerbuna 12, 50009 Zaragoza, Spain; Tel: +34876553774, Fax: +34976762123, E-mail: ferreira@unizar.es

\section{Abbreviations}

AAO, aryl-alcohol oxidase; $\mathrm{AAO}_{\mathrm{ox}}$, oxidized AAO; $\mathrm{AAO}_{\text {red, }}$, reduced AAO; CTC, charge-transfer complex; KIE, kinetic isotope effect; ${ }^{\mathrm{D}}\left({ }^{\mathrm{app}} k_{\text {cat }}\right)$, KIE for apparent $k_{\text {cat }}$ estimated under $\mathrm{O}_{2}$-saturation conditions; ${ }^{\mathrm{D}}\left({ }^{\text {app }} K_{\mathrm{m}(\mathrm{Al})}\right)$, KIE for apparent $K_{\mathrm{m}(\mathrm{Al})}$ estimated under $\mathrm{O}_{2}$-saturation conditions; GMC, glucose-methanol-choline oxidase (oxidoreductase superfamily); $k_{c a t}$, catalytic constant (turnover number); $K_{d}$, dissociation constant; $K_{\mathrm{m}(\mathrm{Al})}$, Michaelis constant for alcohol substrate; $K_{\mathrm{m}(\mathrm{Ox})}$, Michaelis constant for $\mathrm{O}_{2}$ substrate; ${ }^{\text {app }} k_{\mathrm{ox}}$, apparent reoxidation constant; $k_{\text {red }}$, reduction constant; $\mathrm{k}_{1}$ to $\mathrm{k}_{7}$, rates for AAO catalytic cycles/reactions; MM, molecular mechanics; Alc, alcohol; Ald, aldehyde; QM, quantum mechanics

\begin{abstract}
Aryl-alcohol oxidase (AAO) generates $\mathrm{H}_{2} \mathrm{O}_{2}$ for lignin degradation at expenses of benzylic (and other $\pi$-system-containing) primary alcohols, which are oxidized to the corresponding aldehydes. Ligand diffusion on Pleurotus eryngii AAO showed a T-shaped stacking interaction between Tyr92 side-chain and the alcohol substrate at the catalytically-competent
\end{abstract}


position for concerted hydride/proton transfer. Bi-substrate kinetics revealed that reactions with 3-chloro- or 3-fluorobenzyl alcohols (halogen substituents) proceed via a ping-pong mechanism. However, mono- and dimethoxylated substituents (in 4-methoxybenzyl and 3,4dimethoxybenzyl alcohols) switched the mechanism and a ternary complex is formed. This correlated with fully-oxidized AAO during turnover with the above halogenated alcohols, while a reduced fraction (up to $\sim 40 \%$ ) was found for other substrates. Tyr92 contribution to AAO reaction mechanism was investigated by calculation of stacking interaction energies and site-directed mutagenesis. Electron-withdrawing substituents resulted in lower QM stackingenergies between aldehyde and tyrosine side-chain, which would contribute to product release, in agreement with the ping-pong mechanism observed in 3-chloro- and 3fluorobenzyl alcohol kinetics. In contrast, the higher stacking-energies when electron-donor substituents are present would result in $\mathrm{O}_{2}$ reaction with the flavin through a ternary complex, in agreement with the kinetics of methoxylated alcohols. Tyr92 replacement by phenylalanine does not alter the AAO kinetic constants (on 4-methoxybenzyl alcohol), most probably because the stacking interaction is still possible. However, introduction of a tryptophan residue at this position strongly hinders the affinity for the substrate (pre-steady state $K_{\mathrm{d}}$ and steady-state $K_{\mathrm{m}}$ increase by 150 -fold and 75 -fold, respectively) and, therefore the steady-state catalytic efficiency, suggesting that proper stacking is impossible with this bulky residue. The above results confirm the role of Tyr92 in substrate binding, governing the kinetic mechanism in AAO.

\section{Introduction}

Aryl-alcohol oxidases (AAO, EC 1.1.3.7) are extracellular flavoproteins that typically catalyze the oxidative dehydrogenation of polyunsaturated alcohols using molecular oxygen as final electron acceptor and producing hydrogen peroxide [1]. This activity was first reported in Trametes versicolor [2] and has been later described in other white-rot basidiomycetes, mainly responsible for lignin degradation in nature, such as Pleurotus eryngii, Bjerkandera adusta and Phanerochaete chrysosporium [3-7]. Lignin removal is a rate-limiting step for carbon recycling in land ecosystems, playing also a central role in paper pulp manufacture and in the production of chemicals and biofuels from renewable lignocellulosic biomass [8]. The physiological role of AAO in wood-rotting basidiomycetes is to provide a continuous supply of extracellular $\mathrm{H}_{2} \mathrm{O}_{2}$, required as substrate for ligninolytic peroxidases (in white-rot fungi) and as precursor of hydroxyl radical depolymerizing plant polysaccharides (in brown-rot fungi) [1,9].

AAO from Pleurotus eryngii has been intensively investigated [10-14]. This enzyme contains one molecule of non-covanlently bound FAD which acts as two-electron acceptor during the oxidation of a wide range of benzylic and other aromatic and aliphatic polyunsaturated primary alcohols (reductive half-reaction). The oxidative half-reaction, where the FAD hydroquinone is oxidized by $\mathrm{O}_{2}$, closes the catalytic cycle [13-15]. AAO also oxidizes some aromatic aldehydes via their hydrated (gem-diol) forms, suggesting similar mechanisms for alcohol and aldehyde oxidation [16].

The AAO crystal structure (PDB entry 3FIM) confirmed that it shares similar fold topology with other members of the glucose-methanol-choline oxidase (GMC) oxidoreductase superfamily [17], and the role in catalysis of two conserved active-site residues (His502 and His546 in Pleurotus eryngii AAO) was postulated [18]. Reducing substrate diffusion simulations towards AAO active site, previously identified in the crystal structure, suggested an entrance channel next to the Gln395-Thr406 loop [19]. These studies additionally suggested that the enzyme-substrate complex formation, on its catalytically competent form, requires significant displacements of side-chains in the active site environment (including Phe397), as well as the substrate $\pi-\pi$ stacking interaction with Tyr92 
(Fig. 1) [19]. On the contrary, the oxidative substrate migration did not require the above structure reorganization, despite Phe501 was shown to be essential for correct $\mathrm{O}_{2}$ positioning [13].

A hydride transfer reaction assisted by a conserved active site base is the consensus catalytic mechanism in the GMC superfamily [20-26]. An initial mechanistic study on AAO, including analysis of substrate and solvent kinetic isotope effects (KIE), suggested a concerted proton abstraction from the alcohol hydroxyl and hydride transfer from the alcohol $\alpha$-carbon to FAD, without formation of a stable alkoxide intermediate [14]. Later research combining site-directed mutagenesis and quantum mechanics/molecular mechanics (QM/MM) calculations, confirmed the role of His502 as AAO catalytic base [19]. Moreover, the combination of $\mathrm{QM} / \mathrm{MM}$ profiles and solvent KIE data showed that the $\mathrm{O}-\mathrm{H}$ bond cleavage for proton abstraction from the alcohol hydroxyl precedes the $\mathrm{C}_{\alpha}-\mathrm{H}$ bond cleavage for hydride transfer, although both processes are highly coupled. These observations revealed a non-synchronous concerted mechanism for alcohol oxidation by AAO [19]. This finding contrasts with the non-concerted mechanism previously reported for choline and methanol oxidases, which include a stable alkoxide intermediate, and provides an alternative mechanism for alcohol oxidation in the GMC superfamily [27, 28].

In the present study, different aromatic alcohol substrates have been use to complete the description of the AAO overall catalytic cycle and to evaluate the influence of the aromaticstacking interactions on catalysis. This later aim has also been addressed by using some mutants at position Tyr92 to investigate its contribution to the AAO reaction mechanism.

\section{Results}

\section{Influence of alcohol type on AAO kinetic mechanism: Steady-state results}

Double reciprocal plots of the initial rates of AAO reaction with different types of substituted benzyl alcohol substrates at different $\mathrm{O}_{2}$ concentrations yielded two different kinetic patterns. For 3,4-dimethoxybenzyl and 3-chloro-4-methoxybenzyl alcohols, plots were linear and intersected to the left of the Y-axis (below zero with respect to $\mathrm{X}$-axis) (Fig. 2A), as previously reported for 4-methoxybenzyl alcohol and 2-4-hexadien-1-ol [14]. This is indicative of a sequential reaction mechanism involving a ternary complex between AAO and its reducing/oxidizing substrates (Scheme 1, top). On the contrary, a parallel line pattern was obtained for the two other substrates investigated, 3-chlorobenzyl and 3-fluorobenzyl alcohols (Fig. 2B), indicating a ping-pong mechanism (Scheme 1, bottom).

The steady-state kinetic parameters obtained by fitting the experimental data of bisubstrate kinetics to either Eq. 1 or Eq. 2 (describing ternary or ping-pong mechanisms, respectively; see Materials and Methods), are summarized in Table 1. Turnover numbers $\left(k_{\text {cat }}\right)$ calculated under substrate (alcohol and $\mathrm{O}_{2}$ ) saturation conditions showed a similar dependence on the electronic nature of the substituents on the benzenic ring to those previously reported under air atmosphere [15]. The highest $\left(129 \mathrm{~s}^{-1}\right.$ for 4-methoxybenzyl alcohol) and lowest (13 $\mathrm{s}^{-1}$ for 3-fluorobenzyl alcohol) $k_{\text {cat }}$ values were observed in the presence of electron donor and withdrawing substituents, respectively. However, the type of alcohol substrate also had a marked influence on $K_{\mathrm{m}(\mathrm{O} 2)}$, therefore on the AAO affinity for $\mathrm{O}_{2}$. In this way, the enzyme exhibits a considerably higher affinity for $\mathrm{O}_{2}$ when the reaction takes place with alcohols showing a ping-pong mechanism in comparison with those forming a ternary complex: $K_{\mathrm{m}(\mathrm{O} 2)}$ for 3-chlorobenzyl and 3-fluorobenzyl alcohols are up 35 times lower than for 4-methoxybenzyl alcohol. As a consequence, the catalytic efficiency for $\mathrm{O}_{2}$, $k_{\text {cat } /} K_{\mathrm{m}(\mathrm{O} 2)}$, is 3 times higher in the presence of alcohol substrates leading to a ping-pong 
mechanism. Nevertheless, the catalytic efficiency regarding the alcohol substrate, $k_{\mathrm{cat} /} K_{\mathrm{m}(\mathrm{Al})}$, is considerably decreased in the ping-pong mechanism due to both a reduction in $k_{\text {cat }}$ and an increase in $K_{\mathrm{m}(\mathrm{Al})}$ values (Table $\left.\mathbf{1}\right)$.

\section{AAO redox state during turnover}

To further investigate the rate-limiting step during oxidation of the different alcohol substrates, we analyzed the redox state of the FAD cofactor during the steady-state turnover of AAO. Fig. 3 compares the time course of absorbance changes at the $462 \mathrm{~nm}$ maximum during AAO reaction after mixing with an equal volume of each alcohol at saturation concentration under air atmosphere conditions in the stopped-flow equipment. The flavin redox state during the initial steady-state phase, whose time duration varies with the different alcohol substrates, shows the relative rates of AAO reduction (by each alcohol substrate) and its oxidation (by $\mathrm{O}_{2}$ ). For all the assayed substrates AAO is predominantly in the oxidized form during turnover, and the reduced fraction percentage depends on the alcohol type. For 3chloro-4-methoxybenzyl, 3-chlorobenzyl and 3-fluoro-benzyl alcohols, the AAO oxidized form is highly predominant (100-95\%) at the onset of the steady state, indicating that the oxidative-half reaction is much faster than the reductive one. Similar results have been previously observed for 4-methoxybenzyl alcohol ( 80\% of oxidized form) [14]. By contrast, when 3,4-dimethoxybenzyl alcohol was assayed, the percentage of reduced AAO during turnover increased, up to $\sim 40 \%$, indicating that the rates for the reductive and oxidative halfreactions are in this case almost balanced, in agreement with the similar values observed for alcohol and $\mathrm{O}_{2}$ catalytic efficiencies (Table 1).

Regarding the spectral changes during AAO turnover, the first spectra obtained after mixing showed a displacement of the main flavin band (462 $\mathrm{nm})$ and an absorbance increase, reflecting the formation of the oxidized enzyme complex with the alcohol substrate $\left(\mathrm{AAO}_{\mathrm{ox}}{ }^{-}\right.$ Alc). After this initial kinetic event, the results with 3-chloro-4-methoxybenzyl, 4methoxybenzyl and 3,4-dimethoxybenzyl alcohols best fitted a two-step process (Fig. 4A). The first step observed reflects the flavin reduction with concomitant formation of a chargetransfer complex (CTC) between the reduced enzyme and the aldehyde product of the reaction (AAO red -Ald), characterized by a broad band centered at $\sim 550-650 \mathrm{~nm}$. The second observed step involves the spectral perturbation in the initially formed $\mathrm{AAO}_{\text {red }}$-Ald complex with the appearance of a new peak at $490 \mathrm{~nm}$. These events (CTC formation and spectral perturbations) can be related with the presence of $\mathrm{O}_{2}$, since AAO anaerobic reduction by these substrates prevents its formation, as described below (Fig. 5). However, a one-step process is observed with 3-chlorobenzyl and 3-fluorobenzyl alcohols, leading directly from oxidized to fully reduced flavin without stabilizations of any CTC intermediate (Fig. 4B). These turnover results using different substrates, together with the obtained steady-state kinetic constants, provide valuable mechanistic information, which can be correlated with the pre-steady-state studies as described below.

\section{Influence of alcohol type on AAO reductive and oxidative half-reactions}

The time course of AAO reduction by the five alcohol substrates studied was monitored using a stopped-flow spectrophotometer in the $350-900 \mathrm{~nm}$ range under anaerobic conditions. All assayed substrates fully reduced AAO without appearance of any FAD semiquinone intermediate (Fig. 5), consistent with a hydride transfer mechanism as previously reported for 4-methoxybenzyl alcohol and 2,4-hexadien-1-ol [14].

For 3,4-dimethoxybenzyl and 3-chloro-4-methoxybenzyl alcohols the evolution of spectral changes upon AAO reduction was consistent with a two-steps kinetic model $(A \rightarrow B \rightarrow C)$ (Fig. 5A), as previously reported for 4-methoxybenzyl alcohol [14]. The first process $(A \rightarrow B)$ was fast and hyperbolically dependent on substrate concentration, accounting more than the $80 \%$ 
of the reaction amplitude. This step is assigned to substrate oxidation and formation of an $\mathrm{AAO}_{\text {red }}-\mathrm{P}$ complex. The second process $(B \rightarrow C)$ was concentration-independent and too slow (3-5 $\mathrm{s}^{-1}$ ) to be relevant for overall turnover (Table 1). This step might be related to a slow release of the aldehyde product from the reduced AAO active site in the absence of $\mathrm{O}_{2}\left(\mathrm{k}_{3}\right.$ in Scheme 1). Formation of such unstable enzyme-product complexes have also been reported in other flavin-dependent oxidases showing a biphasic reaction course [29, 30]. The observed reduction rates $\left(k_{\text {obs }}\right)$ exhibit a hyperbolic substrate dependence that upon fitting to Eq. 3 or Eq. 4 (see Materials and Methods) are consistent with an essentially irreversible flavin reduction $\left(k_{\text {rev }} \sim 0\right)$. The calculated reduction constants $\left(k_{\text {red }}\right)$ for the AAO reduction by 3,4dimethoxybenzyl and 3-chloro-4-methoxybenzyl alcohols were up to 3-fold faster than their turnover rates, suggesting that the reductive half-reaction is not the limiting step when these alcohols are oxidized.

On the contrary, a one-step model $(A \rightarrow B)$ was the most satisfactory to describe the irreversible AAO reduction by 3-chlorobenzyl and 3-fluorobenzyl alcohols after the initial formation of the $\mathrm{AAO}_{\mathrm{ox}}-\mathrm{Alc}$ complex (Fig. 5B). The $k_{\text {red }}$ values for these two alcohols $(8 \pm 1$ and $6 \pm 1 \mathrm{~s}^{-1}$, respectively) were similar to the above reported turnover rates (Table 1). Cleary indicating that for these substrates the reductive half-reaction is the limiting step in catalysis.

Similary AAO oxidative half-reaction was studied in this case by following the flavin absorbance increase at $462 \mathrm{~nm}$ after mixing the reduced enzyme with buffer containing known $\mathrm{O}_{2}$ concentrations. Independently of the method used to reduce AAO, the rates of flavin reoxidation were linearly dependent on $\mathrm{O}_{2}$ concentration (Fig. S1). When AAO was photo-reduced, the determined second-order rate constant $\left({ }^{\mathrm{app}} k_{\mathrm{ox}}\right.$ of $\left.1.9 \times 10^{4} \mathrm{M}^{-1} \mathrm{~s}^{-1}\right)$ was much higher than the expected by reoxidation of free reduced flavins but clearly inconsistent with the AAO turnover rates. Therefore, we studied the AAO reoxidation reaction after its anaerobic reduction via incubation with a slight excess of different alcohol substrates. For all reducing substrates, the rates of flavin reoxidation were in the range of those reported for others oxidases. The values for second-order rate constants obtained were $7.0 \times 10^{5}, 8.0 \times 10^{5}$ and $8.4 \times 10^{5} \mathrm{M}^{-1} \mathrm{~s}^{-1}$ for 3-chlorobenzyl, 3-chloro-4-methoxybenzyl and 3,4-dimethoxybenzyl alcohol, respectively, similar to those previously reported for 4-methoxybenzyl alcohol $\left(6.7 \times 10^{5} \mathrm{M}^{-1} \mathrm{~s}^{-1}\right)$ [13]. Therefore rates of flavin reoxidation are neither dependent on the nature of the alcohol substrate or on the kinetic mechanism. However, the experimental conditions used do not guarantee the presence of the aldehyde product at the active site during flavin reoxidation (although a slow dissociation is suggested by previous reductive-half reaction experiments).

\section{Stacking stabilization of the substrate in the AAO active site}

AAO oxidizes mainly aromatic and other $\pi$-systems containing conjugated primary alcohols. The aromatic nature of the AAO alcohol substrates pointed to the importance of $\pi$ - $\pi$ stacking in substrate stabilization by AAO. Ligand migration studies showed a T-shaped interaction between Tyr92 and the alcohol substrate in the catalytically-active poses [19]. Starting from this active complex, the Tyr-substrate binding energy was evaluated using ab initio quantum chemistry.

Table 2 shows the T-shaped interaction energies between a tyrosine side-chain and five AAO substrates: 4-methoxybenzyl, 3-chloro-4-methoxybenzyl, 3,4-dimethoxybenzyl, 3chlorobenzyl and 3-fluorobenzyl. For each substrate we modeled both the alcohol and the aldehyde forms. The first clear result is that all the interaction energies are stabilizing, with similar values to those obtained by others [31]. In all cases the stabilization energy is slightly higher for the alcohol reactants than for the aldehyde products. This difference will facilitate the release of the products. Furthermore, important differences are observed for 3chlorobenzyl and 3-fluorobenzyl alcohols. First we observe a lower stacking stabilization for 
the alcoholic form and, second, a larger decrease in the interaction energy with the aldehyde. The significant lower stabilization for the aldehyde product for these two substrates, also establishes a direct correlation between lower $\pi-\pi$ stacking stabilization energies and the pingpong mechanism.

\section{Kinetic properties for Tyr92 variants}

Several site-directed variants of AAO at Tyr92 were prepared to investigate its role on catalysis. The Y92L, Y92F and Y92W variants were purified as oxidized holoproteins, showing an $\mathrm{A}_{280} / \mathrm{A}_{463}$ ratio $(\sim 10)$ similar to the native recombinant enzyme. The absorption spectra of Y92L and Y92F were basically similar to that of the native enzyme, with absorption maxima at 387 and $463 \mathrm{~nm}$, while maxima for the Y92W variant were slightly displaced at $384 \mathrm{~nm}$ and $457 \mathrm{~nm}$ (Fig. S2).

The catalytic properties of the Y92L, Y92F and Y92W AAO variants were determined using 4-methoxybenzyl alcohol as substrate (Table 3). While mutating the tyrosine to phenylalanine only causes a slight increase in $K_{\mathrm{m}(\mathrm{O} 2)}$, its replacing with a leucine produces a decrease in catalityc efficiency for the alcohol substrate (2.6-fold lower) accompanied by $\sim 2-$ fold increases in both $K_{\mathrm{m}(\mathrm{Al})}$ and $K_{\mathrm{d}}$. Finally, the incorporation of a bulkier residue, by the Y92W mutation, causes a strong decrease in catalytic efficiencies for both $\mathrm{O}_{2}$ (6-fold lower) and, particularly, 4-methoxybenzyl alcohol (860-fold lower). Since for the Y92W variant the turnover rate was 10-fold lowered, we conclude that the main effect of the mutation concerns the availability of the alcohol substrate at the AAO active site (with 75-fold higher $K_{\mathrm{m}}$ values). Likewise, the Y92W mutation prevents CTC formation during the enzyme turnover with 4-methoxybenzyl alcohol, also suggesting that substrate accommodation is affected by this mutation (Supplemental Fig. S3).

Fast reaction of the tyrosine variants with 4-methoxybenzyl alcohol was also investigated by anaerobic stopped-flow techniques. The spectral evolution obtained for all of them indicated complete (two electron) enzyme reduction, in agreement with a hydride transfer reaction (Fig. 6). The calculated $k_{\text {red }}$ and $K_{\mathrm{d}}$ agreed with the steady-state $k_{\text {cat }}$ and $K_{\mathrm{m}}$ values (Table 3), indicating that the reductive half-reaction is the rate limiting step in catalysis for these variants.

To investigate whether Tyr92, establishing a stacking interaction with the docked 4methoxybenzyl alcohol (Fig. 1), might contribute to the hydride transfer selectivity previously reported for native AAO [19], we measured the KIE values on apparent steady-state kinetic constants for the Y92L variant using three different isotopically-labelled preparations of this alcohol: $(R)-\left[\alpha-{ }^{2} \mathrm{H}\right]-,(S)-\left[\alpha-{ }^{2} \mathrm{H}\right]-$ and $\left[\alpha-{ }^{2} \mathrm{H}_{2}\right]-4-m e t h o x y b e n z y l$ alcohol (Table 4). The KIE on the apparent turnover values, ${ }^{\mathrm{D}}\left({ }^{\mathrm{app}} k_{\mathrm{cat}}\right)$, slightly decreased $(\sim 20 \%)$ for the $(R)$ and dideuterated forms, being unaffected for the $(S)$ form. However, the KIEs on the apparent catalytic efficiency, ${ }^{\mathrm{D}}\left({ }^{\mathrm{app}} k_{\mathrm{cat}} / K_{\mathrm{m}(\mathrm{Al})}\right)$, for the $(R)$ and, especially, for the dideuterated alcohols were significantly higher for the Y92L variant and, therefore, the ${ }^{\mathrm{D}}\left(K_{\mathrm{m}(\mathrm{Al})}\right)$ values were significantly lower than those of the native AAO. In fact, the small KIE value for ${ }^{\mathrm{D}}\left(K_{\mathrm{m}(\mathrm{Al})}\right)$ for native AAO (deuteration mainly affects the ${ }^{1} \mathrm{H}^{-} /^{2} \mathrm{H}^{-}$abstraction ratio) was absent for the Y92L variant (despite the KIE on turnover was not modified) indicating that Tyr92 contributes to 4methoxybenzyl alcohol binding.

\section{Discussion}

\section{Ping-pong $v s$ ternary mechanism (in AAO and other flavooxidases)}

Comparison with other GMC oxidoreductases, which catalyze the oxidation of alcohol groups in relatively specific reactions, AAO reveals a broad electron donor substrate specificity of AAO oxidizing aromatic and other $\pi$-system substrates with conjugated primary alcohols 
(including benzylic, naphthylic, and aliphatic polyunsaturated alcohols) [5, 15, 32]. These conjugated double bond systems increase the electron availability at the benzylic position enabling hydride abstraction by flavin N5. Moreover, the architecture of the AAO active site prevents the oxidation of secondary alcohols that cannot be accommodated at adequate distances of the catalytic histidine and the above-mentioned flavin N5 atom due to the presence of Phe501 [33].

Here we have performed a detailed study on AAO kinetic mechanism using different alcohol substrates. In all cases the AAO catalytic reaction can be divided into a reductive halfreaction, in which two electrons are transferred via a hydride ion to the oxidized FAD, and an oxidative half-reaction, in which two electrons are transferred from the reduced flavin to $\mathrm{O}_{2}$ yielding hydrogen peroxide. However, based on the results of bisubstrate kinetics analysis with different benzylic alcohols, the overall AAO catalytic cycle seems to be highly influenced by the chemical nature of the substituents in the substrate benzenic ring. For electron-withdrawing substituents (in 3-chloro- and 3-fluorobenzyl alcohols), both halfreactions are independent, where the aldehyde product dissociation is taken place before the $\mathrm{O}_{2}$ (the second substrate) reaction, in a ping-pong steady-state kinetic mechanism (Scheme 1, bottom). On the contrary, a sequential mechanism operates for alcohols with electron-donor substituents (methoxylated benzyl alcohols) in which $\mathrm{O}_{2}$ reacts with the $\mathrm{AAO}_{\text {red-Ald }}$ - complex, forming a ternary complex prior to aldehyde product release (Scheme 1, top).

Vainillyl alcohol oxidase, another versatile flavoenzyme which is able to oxidize aromatic alcohols as AAO, although belonging to a different superfamily, also exhibits a substratedependent overall catalysis [34]. This behavior differs from other fungal GMC oxidoreductases, such as glucose oxidase, pyranose 2-oxidase, cholesterol oxidase and cellobiose dehydrogenase, where ping-pong was reported as the general mechanism [24, 3537]. Interestingly, detailed investigations on pyranose 2-oxidase recently indicated that its steady-state mechanism switches as a function of $\mathrm{pH}[38]$.

\section{Stacking interactions govern AAO catalytic mechanism}

Classical force fields semiquantitatively reproduce T-shape stacking dispersion forces [39] Moreover, high level quantum mechanistic studies pointed out at the importance of T-shaped interactions in molecular stacking, for example in benzene dimer [40]. In AAO, previous ligand migration simulations (using classical force fields) [19] suggested a T-shape substrateTyr92 interaction. Our QM calculations (table 2) confirm such stabilizing interactions for the different substrate (Alc)/product (Ald) benzenic rings. In addition, stacking energies estimated for the different substrate/product correlate fairly well with their electron donating/withdrawing properties (to the ring $\pi$-cloud) of their different substituents. Methoxybenzene nucleophilicity is stronger than benzene due to resonance, increasing the electronic density in the ring. These effects explain why dimethoxylated alcohols/aldehydes have the largest stacking interaction energies. On the other side, for 3-chloro and 3-fluoro substituted alcohols/aldehydes, the halogen acts as an electron withdrawer (inductive effect), resulting in the lowest stabilization energies. Moreover, the slightly different interaction energies for these two halogenated compounds follow the expected trend for the increased electronegativity of the fluoride compared to chloride and thus, stronger inductive electron withdrawing effect. Thus, the lower interaction energies for the two halogenated compared with the methoxylated aldehydes, together with the higher alcohol-aldehyde differential interaction energies, should contribute to the release of the aldehyde product, favoring the ping-pong mechanism. The stacking interactions were intermediate for the 3-chloro-4methoxybenzyl alcohol/aldehyde due to the combination of electron donor and withdrawing substituents. 
Taking together the kinetic data, the stacking energy calculations and the available structural and computational information reported previously, the overall turnover of AAO is consistent with the proposed kinetic model of Scheme 1. Substrate diffusion studies had shown that alcohol and $\mathrm{O}_{2}$ molecules access the AAO active site through the same hydrophobic channel [13, 19]. The alcohol diffusion pathway requires, however, chain displacements and interaction with residues Phe397, Tyr92 and Phe501 to attain the AAO active site, while $\mathrm{O}_{2}$ diffusion does not need any rearrangement. This continuous $\mathrm{O}_{2}$ supply supports the fact that for the sequential mechanism, where the aldehyde product is still bound at the active site upon $\mathrm{O}_{2}$ arrival, some arrangement of the aldehyde product for flavin reoxidation must be required (for more detail see supplemental movie $\mathrm{S} 1$ in $[13,19]$ ). However, the $\mathrm{O}_{2}$ affinity increases when the reaction takes place with alcohols leading to a ping-pong mechanism. This may be caused by prior dissociation of product aldehyde leaving more space at the active site for proper $\mathrm{O}_{2}$ binding.

\section{Rate-limiting step/s in AAO catalysis}

Rapid kinetic experiments provided separate information on the reductive and oxidative halfreactions for each alcohol substrate. For 3-chlorobenzyl and 3-fluorobenzyl alcohols, the reductive half-reaction is the rate determining step in catalysis and responsible of the low efficiency in substrate oxidation.

However, for 3,4-dimethoxybenzyl and 3-chloro-4-methoxybenzyl alcohols, flavin reduction rate is far from being the limiting state. In the first case, the apparent second-order constant estimated from pre-steady kinetic data $\left(k_{\mathrm{red}} / K_{\mathrm{d}}=156 \pm 46 \mathrm{~s}^{-1} \mathrm{mM}^{-1}\right)$ is in agreement with the steady-state alcohol catalytic efficiency obtained $\left(105 \pm 8 \mathrm{~s}^{-1} \mathrm{mM}^{-1}\right)$. In the ternary complex mechanism shown in Scheme 1 (top), $k_{\text {cat }}$ is a combination of catalytic steps in which the 3,4-dimethoxybenzyl alcohol is oxidized to 3,4-dimethoxybenzaldehyde $\left(\mathrm{k}_{2} \approx k_{\mathrm{red}}\right)$ and the product is released $\left(\mathrm{k}_{5}\right)\left(k_{\mathrm{cat}}=\mathrm{k}_{2} \mathrm{k}_{5} /\left(\mathrm{k}_{2}+\mathrm{k}_{5}\right)\right)$. The estimated value for $\mathrm{k}_{5}\left(101 \mathrm{~s}^{-1}\right)$ would be indicative of a partially rate-limiting step for catalysis, suggesting that other kinetic step (after hydride transfer and before product release) also contributes to limiting the reaction rate. This step could be the flavin reoxidation, as suggested by the $40 \%$ AAO reduction during enzyme turnover when this substrate was assayed, showing similar values for alcohol and $\mathrm{O}_{2}$ catalytic efficiencies.Finally, for 3-chloro-4-methoxybenzyl alcohol oxidation, the estimated $\mathrm{k}_{5}$ value $\left(42 \mathrm{~s}^{-1}\right)$ suggests thatproduct release could be the rate-limiting step (Scheme 1, top). In fact, previous studies on 3-chloro-4-methoxybenzyl alcohol oxidation by AAO indicate that a fraction of the aldehyde formed was oxidized to the corresponding acid, rather than being released from the active site [16].

Regarding the oxidative half-reaction, reoxidation of AAO, previously reduced by different alcohols, yielded bimolecular rate constants $\left(7.0 \times 10^{5}-8.4 \times 10^{5} \mathrm{M}^{-1} \mathrm{~s}^{-1}\right)$ in the typical range described for other flavooxidases, $\sim 3$ orders of magnitude larger that the values reported for non-enzymatic reoxidation of free flavins $\left(250 \mathrm{M}^{-1} \mathrm{~s}^{-1}\right)$, and $\sim 40$-fold faster than found for photo-reduced AAO [41, 42]. The latter observation is consistent with enhanced $\mathrm{O}_{2}$ reactivity of AAO when the conserved active site His502 is protonated during the oxidative halfreaction, reducing the singlet/triplet energy gap [43]. The importance of a positively charged group for $\mathrm{O}_{2}$ activation has been reported for flavoprotein oxidases [41, 44].

\section{Tyr92 role in AAO catalysis}

Finally, the kinetic data obtained in the present work for the Tyr92 variants strongly suggest that this residue is involved in the alcohol substrate stabilization at AAO active site. The Y92F variant exhibits similar rates for 3-methoxybenzyl alcohol oxidation than the native enzyme, while the leucine and tryptophan substitutions mainly alter alcohol substrate affinity. In the case of the Y92W variant, substrate binding results significantly weakened with 76 and 
150 fold higher $K_{\mathrm{m}}$ and $K_{\mathrm{d}}$, respectively, while the changes on the affinity constants for the Y92L variant were modest (suggesting that some stacking is still possible). In a previous study, Tyr92 substitution with alanine (fully removing the stacking interaction) did not yield active enzyme, supporting the involvement of the above-mentioned $\pi-\pi$ interaction in AAO catalysis [18]. Interestingly is that Tyr92 is not conserved in the GMC oxidoreductase superfamily. However, tyrosine, phenylalanine and leucine residues are frequently found at this position in the putative AAO sequences from different basidiomycetes genomes (Fig. 7). This suggests that stacking might be a common catalytic strategy for alcohol substrate oxidation in AAO enzymes.

In conclusion the study of the overall catalytic cycle of AAO presented herein shows that stacking stabilization interaction of the aromatic substrate/product by active-site Tyr92 governs AAO catalysis, switching between the ping-pong and the ternary mechanism depending on the stacking stabilization energies. The importance of Tyr92 for alcohol substrate binding was also evidenced by site-directed mutagenesis, kinetics and KIE studies suggesting a common role in other AAO proteins where this residue is conserved or replaced by others which are able to stablish similar stacking interactions.

\section{Materials and methods}

\section{Chemicals}

4-Methoxybenzyl, 3,4-dimethoxybenzyl (veratryl), 3-chlorobenzyl, and 3-fluorobenzyl alcohols were purchased from Sigma-Aldrich (St. Louis, MO, USA). 3-Chloro-4methoxybenzyl alcohol, $(R)-\left[\alpha-{ }^{2} \mathrm{H}\right]-4-m e t h o x y b e n z y l$ alcohol, $(S)-\left[\alpha-{ }^{2} \mathrm{H}\right]-4$-methoxybenzyl alcohol and $\left[\alpha-{ }^{2} \mathrm{H}_{2}\right]-4-$ methoxybenzyl alcohol were synthesized at the Instituto de Ciencia de Materiales de Aragón (CSIC-UZ, Zaragoza, Spain).

\section{Protein production and purification}

Native AAO from $P$. eryngii was obtained by expression in E. coli of the mature AAO cDNA (GenBank AF064069), followed by in vitro activation in the presence of the FAD cofactor, and purification by ion-exchange chromatography as described previously [45]. Mutated variants were prepared using the QuikChange site-directed mutagenesis kit (Stratagene). For the PCR reactions, the cDNA cloned into the pFLAG1 vector was used as template, and the following oligonucleotides (direct sequences) bearing mutations (italics) as primers: i) Y92L, 5'- GGG TCT AGC TCT GTT CAC CTC ATG GTC ATG ATG CG-3'; ii) Y92F, 5'- GGG TCT AGC TCT GTT CAC TTC ATG GTC ATG ATG CG-3'; and iii) Y92W, 5'-GGG TCT AGC TCT GTT CAC TGG ATG GTC ATG ATG CG-3'. Mutations were confirmed by sequencing (GS-FLX sequencer from Roche) and the mutated variants were obtained as described for recombinant AAO. Naturally-oxidized AAO concentration was determined using the molar absorbances of native AAO and of its Y92L, Y92F and Y92W variants $\left(\varepsilon_{463}\right.$ $11050 \mathrm{M}^{-1} \mathrm{~cm}^{-1}, \varepsilon_{463} 11240 \mathrm{M}^{-1} \mathrm{~cm}^{-1}, \varepsilon_{463} 10044 \mathrm{M}^{-1} \mathrm{~cm}^{-1}, \varepsilon_{457} 10693 \mathrm{M}^{-1} \mathrm{~cm}^{-1}$, respectively) calculated by heat denaturation and estimation of the free FAD released $\left(\varepsilon_{450} 11300 \mathrm{M}^{-1} \cdot \mathrm{cm}^{-1}\right)$ [45].

\section{Steady-state kinetic measurements}

Steady-state kinetics measurements were spectrophotometrically monitored by following the oxidation of the alcohol substrate to the corresponding aldehyde as previously described [15]. Two-substrate steady-state kinetics measurements were performed by simultaneously varying the concentrations of alcohol substrate and $\mathrm{O}_{2}$ in $0.1 \mathrm{M}$ phosphate buffer, $\mathrm{pH} 6$, at $12{ }^{\circ} \mathrm{C}$. The reactions were carried out in a screw-cap cuvette where the buffer solution was first equilibrated at the desired concentration of $\mathrm{O}_{2}\left(61,152,319,668\right.$ and $1520 \mu \mathrm{M}$ at $\left.12{ }^{\circ} \mathrm{C}\right)$ by 
bubbling with the appropriate $\mathrm{O}_{2} / \mathrm{N}_{2}$ gas mixture for 10-15 min. Then, reactions were started by the addition of the alcohol substrate (around 5-10 $\mu \mathrm{L}$ ) and AAO $(5 \mu \mathrm{L}, 0.03 \mu \mathrm{M}$ final concentration) into a reaction mixture with a final volume of $1.5 \mathrm{~mL}$. Initial rates were calculated during the linear phase of alcohol oxidation to corresponding aldehyde, and analyzed by fitting to Eq. 1 or Eq. 2 describing ternary complex and ping-pong mechanisms, respectively:

$$
\begin{aligned}
& \frac{v}{e}=\frac{k_{\mathrm{cat}} A B}{K_{\mathrm{m}(\mathrm{Ox})} A+K_{\mathrm{m}(\mathrm{Al})} B+A B+K_{\mathrm{i}(\mathrm{Al})} K_{\mathrm{m}(\mathrm{Ox})}} \\
& \frac{v}{e}=\frac{k_{c a t} A B}{K_{\mathrm{m}(\mathrm{Ox})} A+K_{\mathrm{m}(\mathrm{Al})} B+A B}
\end{aligned}
$$

where $v$ represents the observed initial rate, $e$ is the enzyme concentration, $k_{\text {cat }}$ is the maximal turnover, $A$ is the alcohol substrate concentration , $B$ is the $\mathrm{O}_{2}$ concentration, $K_{\mathrm{m}(\mathrm{Al})}$ and $K_{\mathrm{m}(\mathrm{Ox})}$ are the Michaelis constants for alcohol and $\mathrm{O}_{2}$, respectively, and $K_{\mathrm{i}(\mathrm{Al})}$ is the alcohol dissociation constant .

\section{Stopped-flow measurements: Enzyme turnover and pre-steady-state kinetics}

Stopped-flow experiments were carried out on an Applied Photophysics SX17. MV spectrophotometer (Applied Photophysics Ltd.) using the SX18.MV or the Xscan softwares for experiments with single wavelength or photodiode-array detection, respectively. For enzyme-monitored turnover experiments, air-saturated enzyme and substrate solutions were mixed, and the evolution of the flavin redox state was monitored in the range of 350-900 nm.

Reductive half-reaction studies were performed under anaerobic conditions. Tonometers containing enzyme and substrate solutions were made anaerobic by successive evacuation and flushing with argon. These solutions also contained glucose $(10 \mathrm{mM})$ and glucose oxidase (10 $\mathrm{U} \cdot \mathrm{mL}^{-1}$ ) to ensure anaerobiosis. Drive syringes in the stopped-flow apparatus were made anaerobic by sequentially passing a sodium dithionite solution and $\mathrm{O}_{2}$-free buffer [46]. Measurements were carried out in $0.1 \mathrm{M}$ phosphate buffer, $\mathrm{pH} 6$, at $12{ }^{\circ} \mathrm{C}$. All given concentrations are those after mixing an equal volume of substrate and enzyme (i.e. final concentrations). Spectral evolution was studied by global analysis and numerical integration methods using the Pro-K software (Applied Photophysics Ltd.). Observed rate constant $\left(k_{\mathrm{obs}}\right)$ from traces recorded at $462 \mathrm{~nm}$ were calculated from exponential fits. Rate constants were obtained by a nonlinear fitting of $k_{\mathrm{obs}}$ at different substrate concentrations to either Eq. 3 or Eq. 4:

$$
\begin{aligned}
& k_{\mathrm{obs}}=\frac{k_{\mathrm{red}} A}{K_{\mathrm{d}}+A} \\
& k_{\mathrm{obs}}=\frac{k_{\mathrm{red}} A}{K_{\mathrm{d}}+A}+k_{\mathrm{rev}}
\end{aligned}
$$

where $k_{\mathrm{obs}}$ is the observed rate for reduction of the enzyme at a given alcohol concentration, $k_{\text {red }}$ and $k_{\text {rev }}$ are the limiting rates for hydride transfer from the substrate to the flavin and for the reverse reaction, respectively, at saturating substrate concentrations and $K_{\mathrm{d}}$ is the dissociation constant for the enzyme-substrate complex.

Rate constants for the oxidative half-reaction were measured at $12{ }^{\circ} \mathrm{C}$ in $0.1 \mathrm{M}$ phosphate buffer by monitoring the absorbance increase at $462 \mathrm{~nm}$ upon mixing the previously anaerobically reduced enzyme with buffer equilibrated equilibrated at different $\mathrm{O}_{2}$ concentrations. The enzyme was reduced either by mixing the oxidized protein under anaerobic conditions with a 1.2 fold-excess of the corresponding alcohol substrate or by photoreduction in the presence of $2 \mathrm{mM}$ 5-deazariboflavin and $3 \mathrm{mM}$ EDTA [15]. The 
apparent second-order rate constants for the oxidative half-reaction $\left(k_{\mathrm{ox}}\right)$ were determined as a function of the $\mathrm{O}_{2}$ concentrations and calculated using Eq. 5, where the $k_{\mathrm{obs}}$ is the experimentally observed rate constant associated for flavin oxidation at any given $\mathrm{O}_{2}$ concentration

$$
k_{\text {obs }}={ }^{\text {app }} k_{\text {ox }}\left[\mathrm{O}_{2}\right]
$$

\section{KIE in $p$-methoxybenzyl alcohol oxidation}

The substrate KIEs due to $p$-methoxybenzyl alcohol $\alpha$-deuteration $(R, S$ and dideuterated forms) on apparent steady-state kinetic constants were measured in $\mathrm{O} 2$-saturated $(0.1279$ $\mathrm{mM}) 0.1 \mathrm{M}$ phosphate buffer, $\mathrm{pH} 6.0$ at $25^{\circ} \mathrm{C}$, were and calculated by fitting the initial rates to Eq. 6, which describes a mechanism with separate isotope effects on $k_{\text {cat }}$ and $k_{\text {cat }} / K_{\mathrm{m}}$; where $S$ is substrate concentration, $F_{i}$ is the atom fraction of deuterium label in the substrate $(0.98$ in the present case) and $E_{k_{\text {cat }} / K_{\mathrm{m}}}$ and $E_{k_{\text {cat }}}$ are the isotope effect minus 1 in the two kinetic constants [47].

$$
\frac{v}{e}=\frac{k_{\text {cat }} S}{K_{\mathrm{m}}\left(1+F_{i} E_{\frac{k_{\text {cat }}}{K_{\mathrm{m}}}}\right)+S\left(1+F_{i} E_{k_{\text {cat }}}\right)}
$$

\section{QM calculations}

Second-order Moller-Plesset (MP2) [48] QM calculations were performed using the Gaussian03 program [49]. Triple-zeta split-valence basis sets with polarization orbitals (6$311 \mathrm{G}^{*}$ ) were used throughout. The structures of the T-shaped complexes between a model tyrosine side-chain and the 4-methoxybenzyl alcohol were optimized using MP2 in gas phase and the most stable structure was selected. To isolate the stacking interactions and avoid distortions from the gas phase minimization for the remaining complexes, the $\mathrm{H}$ atom in meta position of the methoxybenzyl structure was replaced by a chloride or a methoxy group. The 3-chlorobenzyl alcohol was obtained by removing the para methoxyl from the 3-chloro-4methoxybenzyl while the 3-fluorobenzyl alcohol structure was obtained by a chloride to fluoride change in 3-chlorobenzyl alcohol. At this point, the benzene rings are maintained frozen and all substituents are optimized. This procedure was done for both the alcohol and aldehyde forms. Binding energies were approximated from the interaction energies: AB$(\mathrm{A}+\mathrm{B})$ for all complexes and basis-set-superposition-error (BSSE) corrections [50] were taken into account at the same level of theory. Several studies on substituted benzene dimers have shown that this level of theory is capable of a qualitative description of differences in stabilization energies [51].

\section{Acknowledgements}

This work was supported by the BIO2013-42978-P (to MM), BIO2011-26694 (to ATM), "Juan de la Cierva" (to FL) and CTQ2010-18123 (to VG) Grants of the Spanish Ministry of Economy and Competitiveness (MINECO) and by the INDOX (KBBE-2013-7-613549, to ATM) and PELE (ERC-2009-Adg 25027, to VG) European projects.

\section{Author contributions}

All of the authors performed the research and discussed the results obtained. P. Ferreira conceived the study, performed some kinetics studies, produced figures, and wrote the paper, A. Hernandez-Ortega designed and constructed the mutations and performed some kinetic studies. F. Lucas, B. Kenneth and V. Guallar performed the computational part of the work and wrote the corresponding sections of the paper. J. Carro produced and characterized the 
protein variants and produce figures. B. Herguedas performed some kinetic studies. AT Martinez and M Medina contributed to integrate the study and write the paper.

\section{References}

1 Hernandez-Ortega, A., Ferreira, P. and Martinez, A. T. (2012) Fungal aryl-alcohol oxidase: a peroxide-producing flavoenzyme involved in lignin degradation. Appl Microbiol Biotechnol. 93, 1395-1410

2 Farmer, V. C., Henderson, M. E. and Russell, J. D. (1960) Aromatic-alcohol-oxidase activity in the growth medium of Polystictus versicolor. Biochem J. 74, 257-262

3 Bourbonnais, R. and Paice, M. G. (1988) Veratryl alcohol oxidases from the lignindegrading basidiomycete Pleurotus sajor-caju. Biochem J. 255, 445-450

4 Sannia, G., Limongi, P., Cocca, E., Buonocore, F., Nitti, G. and Giardina, P. (1991) Purification and characterization of a veratryl alcohol oxidase enzyme from the lignin degrading basidiomycete Pleurotus ostreatus. Biochim Biophys Acta. 1073, 114-119

5 Guillen, F., Martinez, A. T. and Martinez, M. J. (1992) Substrate specificity and properties of the aryl-alcohol oxidase from the ligninolytic fungus Pleurotus eryngii. Eur $\mathbf{J}$ Biochem. 209, 603-611

6 Asada, Y., Watanabe, A., Ohtsu, Y. and Kuwahara, M. (1995) Purification and characterization of an aryl-alcohol oxidase from the lignin-degrading basidiomycete Phanerochaete chrysosporium. Biosci.Biotechnol.Biochem. . 59, 1339-1341

7 Muheim, A., Waldner, R., Leisola, M. S. A. and Fiechter, A. (1990) An extracellular aryl-alcohol oxidase from the white-rot fungus Bjerkandera adusta. Enzyme Microb.Technol. 12, 204-209

8 Ruiz-Duenas, F. J. and Martinez, A. T. (2009) Microbial degradation of lignin: how a bulky recalcitrant polymer is efficiently recycled in nature and how we can take advantage of this. Microb Biotechnol. 2, 164-177

9 Martinez, A. T., Speranza, M., Ruiz-Duenas, F. J., Ferreira, P., Camarero, S., Guillen, F., Martinez, M. J., Gutierrez, A. and del Rio, J. C. (2005) Biodegradation of lignocellulosics: microbial, chemical, and enzymatic aspects of the fungal attack of lignin. Int Microbiol. 8, 195-204

10 Hernandez-Ortega, A., Ferreira, P. and Martinez, A. T. Fungal aryl-alcohol oxidase: a peroxide-producing flavoenzyme involved in lignin degradation. Appl Microbiol Biotechnol. 93, 1395-1410

11 Hernandez-Ortega, A., Lucas, F., Ferreira, P., Medina, M., Guallar, V. and Martinez, A. T. Role of active site histidines in the two half-reactions of the aryl-alcohol oxidase catalytic cycle. Biochemistry. 51, 6595-6608

12 Hernandez-Ortega, A., Ferreira, P., Merino, P., Medina, M., Guallar, V. and Martinez, A. T. Stereoselective hydride transfer by aryl-alcohol oxidase, a member of the GMC superfamily. Chembiochem. 13, 427-435

13 Hernandez-Ortega, A., Lucas, F., Ferreira, P., Medina, M., Guallar, V. and Martinez, A. T. (2011) Modulating O2 reactivity in a fungal flavoenzyme: involvement of aryl-alcohol oxidase Phe-501 contiguous to catalytic histidine. J Biol Chem. 286, 41105-41114

14 Ferreira, P., Hernandez-Ortega, A., Herguedas, B., Martinez, A. T. and Medina, M. (2009) Aryl-alcohol oxidase involved in lignin degradation: a mechanistic study based on steady and pre-steady state kinetics and primary and solvent isotope effects with two alcohol substrates. J Biol Chem. 284, 24840-24847

15 Ferreira, P., Medina, M., Guillen, F., Martinez, M. J., Van Berkel, W. J. and Martinez, A. T. (2005) Spectral and catalytic properties of aryl-alcohol oxidase, a fungal flavoenzyme acting on polyunsaturated alcohols. Biochem J. 389, 731-738 
16 Ferreira, P., Hernandez-Ortega, A., Herguedas, B., Rencoret, J., Gutierrez, A., Martinez, M. J., Jimenez-Barbero, J., Medina, M. and Martinez, A. T. (2009) Kinetic and chemical characterization of aldehyde oxidation by fungal aryl-alcohol oxidase. Biochem $\mathrm{J}$. 425, 585-593

17 Fernandez, I. S., Ruiz-Duenas, F. J., Santillana, E., Ferreira, P., Martinez, M. J., Martinez, A. T. and Romero, A. (2009) Novel structural features in the GMC family of oxidoreductases revealed by the crystal structure of fungal aryl-alcohol oxidase. Acta Crystallogr D Biol Crystallogr. 65, 1196-1205

18 Ferreira, P., Ruiz-Duenas, F. J., Martinez, M. J., van Berkel, W. J. and Martinez, A. T. (2006) Site-directed mutagenesis of selected residues at the active site of aryl-alcohol oxidase, an $\mathrm{H} 2 \mathrm{O} 2$-producing ligninolytic enzyme. FEBS J. 273, 4878-4888

19 Hernandez-Ortega, A., Borrelli, K., Ferreira, P., Medina, M., Martinez, A. T. and Guallar, V. (2011) Substrate diffusion and oxidation in GMC oxidoreductases: an experimental and computational study on fungal aryl-alcohol oxidase. Biochem J. 436, 341350

20 Hecht, H. J., Kalisz, H. M., Hendle, J., Schmid, R. D. and Schomburg, D. (1993) Crystal-Structure of Glucose-Oxidase from Aspergillus-Niger Refined at 2 .3 Angstrom Resolution. Journal of Molecular Biology. 229, 153-172

21 Yue, Q. K., Kass, I. J., Sampson, N. S. and Vrielink, A. (1999) Crystal structure determination of cholesterol oxidase from Streptomyces and structural characterization of key active site mutants. Biochemistry. 38, 4277-4286

22 Lario, P. I., Sampson, N. and Vrielink, A. (2003) Sub-atomic resolution crystal structure of cholesterol oxidase: what atomic resolution crystallography reveals about enzyme mechanism and the role of the FAD cofactor in redox activity. J Mol Biol. 326, 1635-1650

23 Kass, I. J. and Sampson, N. S. (1998) Evaluation of the role of His447 in the reaction catalyzed by cholesterol oxidase. Biochemistry. 37, 17990-18000

24 Hallberg, B. M., Henriksson, G., Pettersson, G., Vasella, A. and Divne, C. (2003) Mechanism of the reductive half-reaction in cellobiose dehydrogenase. J Biol Chem. 278, $7160-7166$

25 Hallberg, B. M., Henriksson, G., Pettersson, G. and Divne, C. (2002) Crystal structure of the flavoprotein domain of the extracellular flavocytochrome cellobiose dehydrogenase. $\mathrm{J}$ Mol Biol. 315, 421-434

26 Kass, I. J. and Sampson, N. S. (1998) The importance of GLU361 position in the reaction catalyzed by cholesterol oxidase. Bioorg Med Chem Lett. 8, 2663-2668

27 Menon, V., Hsieh, C. T. and Fitzpatrick, P. F. (1995) Substituted Alcohols as Mechanistic Probes of Alcohol Oxidase. Bioorganic Chemistry. 23, 42-53

28 Fan, F. and Gadda, G. (2005) On the catalytic mechanism of choline oxidase. J Am Chem Soc. 127, 2067-2074

29 Pollegioni, L., Wels, G., Pilone, M. S. and Ghisla, S. (1999) Kinetic mechanisms of cholesterol oxidase from Streptomyces hygroscopicus and Brevibacterium sterolicum. Eur J Biochem. 264, 140-151

30 Prongjit, M., Sucharitakul, J., Wongnate, T., Haltrich, D. and Chaiyen, P. (2009) Kinetic mechanism of pyranose 2-oxidase from trametes multicolor. Biochemistry. 48, 41704180

31 Mutasem, O. S. and Sherrill, D. C. (2003) Unexpected Substituent Effects in Face-toFace $\pi$-Stacking Interactions. J. Phys. Chem. 107, 8377-8379

32 Romero, E., Ferreira, P., Martinez, A. T. and Martinez, M. J. (2009) New oxidase from Bjerkandera arthroconidial anamorph that oxidizes both phenolic and nonphenolic benzyl alcohols. Biochim Biophys Acta. 1794, 689-697 
33 Hernandez-Ortega, A., Ferreira, P., Merino, P., Medina, M., Guallar, V. and Martinez, A. T. (2012) Stereoselective hydride transfer by aryl-alcohol oxidase, a member of the GMC superfamily. Chembiochem. 13, 427-435

34 Fraaije, M. W. and van Berkel, W. J. (1997) Catalytic mechanism of the oxidative demethylation of 4-(methoxymethyl)phenol by vanillyl-alcohol oxidase. Evidence for formation of a p-quinone methide intermediate. J Biol Chem. 272, 18111-18116

35 Gibson, Q. H., Swoboda, B. E. and Massey, V. (1964) Kinetics and Mechanism of Action of Glucose Oxidase. J Biol Chem. 239, 3927-3934

36 Artolozaga, M. J., Kubatova, E., Volc, J. and Kalisz, H. M. (1997) Pyranose 2-oxidase from Phanerochaete chrysosporium--further biochemical characterisation. Appl Microbiol Biotechnol. 47, 508-514

37 Kujawa, M., Ebner, H., Leitner, C., Hallberg, B. M., Prongjit, M., Sucharitakul, J., Ludwig, R., Rudsander, U., Peterbauer, C., Chaiyen, P., Haltrich, D. and Divne, C. (2006) Structural basis for substrate binding and regioselective oxidation of monosaccharides at $\mathrm{C} 3$ by pyranose 2-oxidase. J Biol Chem. 281, 35104-35115

38 Rungsrisuriyachai, K. and Gadda, G. (2009) A pH switch affects the steady-state kinetic mechanism of pyranose 2-oxidase from Trametes ochracea. Arch Biochem Biophys. 483, 10-15

39 Sherrill, C. D., Takatani, T. and Hohenstein, E. G. (2009) An assessment of theoretical methods for nonbonded interactions: comparison to complete basis set limit coupled-cluster potential energy curves for the benzene dimer, the methane dimer, benzene-methane, and benzene-H2S. J Phys Chem A. 113, 10146-10159

40 Dinadayalane, T. C. and Leszczynski, J. (2009) Geometries and stabilities of various configurations of benzene dimer: details of novel V-shaped structure revealed. Structural Chemistry. 20, 11-20

41 Mattevi, A. (2006) To be or not to be an oxidase: challenging the oxygen reactivity of flavoenzymes. Trends Biochem Sci. 31, 276-283

42 Massey, V. (1994) Activation of molecular oxygen by flavins and flavoproteins. J Biol Chem. 269, 22459-22462

43 Hernandez-Ortega, A., Lucas, F., Ferreira, P., Medina, M., Guallar, V. and Martinez, A. T. (2012) Role of active site histidines in the two half-reactions of the aryl-alcohol oxidase catalytic cycle. Biochemistry. 51, 6595-6608

44 Gadda, G. (2012) Oxygen activation in flavoprotein oxidases: the importance of being positive. Biochemistry. 51, 2662-2669

45 Ruiz-Duenas, F. J., Ferreira, P., Martinez, M. J. and Martinez, A. T. (2006) In vitro activation, purification, and characterization of Escherichia coli expressed aryl-alcohol oxidase, a unique H2O2-producing enzyme. Protein Expr Purif. 45, 191-199

46 Medina, M., Martinez-Julvez, M., Hurley, J. K., Tollin, G. and Gomez-Moreno, C. (1998) Involvement of glutamic acid 301 in the catalytic mechanism of ferredoxin-NADP+ reductase from Anabaena PCC 7119. Biochemistry. 37, 2715-2728

47 Sobrado, P., Daubner, S. C. and Fitzpatrick, P. F. (2001) Probing the relative timing of hydrogen abstraction steps in the flavocytochrome b2 reaction with primary and solvent deuterium isotope effects and mutant enzymes. Biochemistry. 40, 994-1001

48 Wilson, A. K., Woon, D. E., Peterson, K. A. and H, D. T. (1999) Gaussian basis sets for use in correlated molecular calculations. IX. The atoms gallium through krypton. J. Phys. Chem. 110, 7667-7676

49 Frisch, M. J., Trucks, G. W., Schlegel, H. B., Scuseria, G. E., Robb, M. A., Cheeseman, J. R., Montgomery, J. J. A., Vreven, T., Kudin, K. N., Burant, J. C., Millam, J. M., Iyengar, S. S., Tomasi, J., Barone, V., Mennucci, B., Cossi, M., Scalmani, G., Rega, N., Petersson, G. A., Nakatsuji, H., Hada, M., Ehara, M., Toyota, K., Fukuda, R., Hasegawa, J., 
Ishida, M., Nakajima, T., Honda, Y., Kitao, O., Nakai, H., Klene, M., Li, X., Knox, J. E., Hratchian, H. P., Cross, J. B., Bakken, V., Adamo, C., Jaramillo, J., Gomperts, R., Stratmann, R. E., Yazyev, O., Austin, A. J., Cammi, R., Pomelli, C., Ochterski, J. W., Ayala, P. Y., Morokuma, K., Voth, G. A., Salvador, P., Dannenberg, J. J., Zakrzewski, V. G., Dapprich, S., Daniels, A. D., Strain, M. C., Farkas, O., Malick, D. K., Rabuck, A. D., Raghavachari, K., Foresman, J. B., Ortiz, J. V., Cui, Q., Baboul, A. G., Clifford, S., Cioslowski, J., Stefanov, B. B., Liu, G., Liashenko, A., Piskorz, P., Komaromi, I., Martin, R. L., Fox, D. J., Keith, T., AlLaham, M. A., Peng, C. Y., Nanayakkara, A., Challacombe, M., Gill, P. M. W., Johnson, B., Chen, W., Wong, M. W., Gonzalez, C. and Pople, J. A. (2004) Gaussian 03, Revision C.02. Gaussian, Inc., Wallingford CT

50 Boys, S. F. and Bernardi, F. (1970) The calculation of small molecular interactions by the differences of separate total energies. Some procedures with reduced errors. Molecular Physics. 19, 553-566

51 Lee, E. C., Kim, D., Jurecka, P., Tarakeshwar, P., Hobza, P. and Kim, K. S. (2007) Understanding of assembly phenomena by aromatic-aromatic interactions: benzene dimer and the substituted systems. J Phys Chem A. 111, 3446-3457

52 Borrelli, K. W., Cossins, B. and Guallar, V. Exploring hierarchical refinement techniques for induced fit docking with protein and ligand flexibility. J Comput Chem. 31, $1224-1235$ 
Table 1. Kinetics parameters for the steady-state reaction and for the pre-steady-state reductive half-reaction of AAO with five different alcohol substrates

\begin{tabular}{lcccccccc}
\hline & \multicolumn{3}{c}{ Steady-state turnover } & & \multicolumn{2}{c}{ Reductive half-reaction } \\
\cline { 2 - 5 } Alcohol substrate & $\begin{array}{c}k_{\mathrm{cat}} \\
\left(\mathrm{s}^{-1}\right)\end{array}$ & $\begin{array}{c}K_{\mathrm{m}(\mathrm{Al})} \\
(\mu \mathrm{M})\end{array}$ & $\begin{array}{c}k_{\mathrm{cat}} / K_{\mathrm{m}(\mathrm{Al})} \\
\left(\mathrm{s}^{-1} \mathrm{mM}^{-1}\right)\end{array}$ & $\begin{array}{c}K_{\mathrm{m}(\mathrm{Ox})} \\
(\mu \mathrm{M})\end{array}$ & $\begin{array}{c}k_{\mathrm{cat}} / K_{\mathrm{m}(\mathrm{Ox})} \\
\left(\mathrm{s}^{-1} \mathrm{mM}^{-1}\right)\end{array}$ & $\begin{array}{c}k_{\mathrm{red}} \\
\left(\mathrm{s}^{-1}\right)\end{array}$ & $\begin{array}{c}K_{\mathrm{d}} \\
(\mu \mathrm{M})\end{array}$ \\
\hline 4-Methoxybenzyl & $129 \pm 5$ & $25 \pm 3$ & $5160 \pm 650$ & $348 \pm 36$ & $371 \pm 41$ & $115 \pm 3$ & $31 \pm 2$ \\
3-Chloro-4-methoxybenzyl & $29 \pm 1$ & $8 \pm 1$ & $3630 \pm 470$ & $214 \pm 30$ & $136 \pm 20$ & $95 \pm 3$ & $71 \pm 10$ \\
3,4-Dimethoxybenzyl & $57 \pm 1$ & $543 \pm 39$ & $105 \pm 8$ & $119 \pm 11$ & $479 \pm 45$ & $131 \pm 10$ & $838 \pm 242$ \\
3-Chlorobenzyl & $13 \pm 1$ & $62 \pm 1$ & $210 \pm 4$ & $10 \pm 1$ & $1300 \pm 130$ & $8 \pm 1$ & $58 \pm 2$ \\
3-Fluorobenzyl & $9 \pm 1$ & $164 \pm 3$ & $56 \pm 1$ & $7 \pm 1$ & $1400 \pm 160$ & $6 \pm 1$ & $180 \pm 7$ \\
\hline
\end{tabular}

Measurements were performed in $0.1 \mathrm{M}$ phosphate buffer, $\mathrm{pH} 6$, at $12^{\circ} \mathrm{C}$. Steady-state kinetic constants were determined by varying the concentrations of both alcohol and $\mathrm{O}_{2}$, and calculated by fitting to Eq. 1 (4-methoxybenzyl, 3-chloro-4-methoxybenzyl and 3,4dimethoxybenzyl alcohols) or Eq. 2 (3-chlorobenzyl and 3-fluorobenzyl alcohols) describing either a ternary or ping-pong mechanism, respectively (see Materials and Methods). The pre-steady state observed reduction rate constants were fitted to Eq. 3. Means and standard desviations are provided ${ }^{1}$ Data from Ferreira et al. [14] 
Table 2: T-shaped stacking energies between five AAO alcohol substrates and their corresponding aldehydes with a tyrosine side-chain, and differences between the two calculated energies (in $\mathrm{Kcal} \cdot \mathrm{mol}^{-1}$ )

\begin{tabular}{lccc}
\hline & Alcohols (Alc) & Aldehyde (Ald) & Difference (Alc-Ald) \\
\hline 4-Methoxybenzyl & -3.27 & -2.83 & -0.44 \\
3-Chloro-4-methoxybenzyl & -3.16 & -3.00 & -0.16 \\
3,4-Dimethoxybenzyl & -4.11 & -3.62 & -0.49 \\
3-Chlorobenzyl & -2.77 & -1.96 & -0.81 \\
3-Fluorobenzyl & -2.67 & -1.81 & -0.86 \\
\hline
\end{tabular}

T-shaped stacking energies between Tyr and alcohol (Alc) or aldehyde (Ald) were calculated as described in the Materials and Methods section. Stacking energy difference between alcohol and substrate (Alc-Ald) is shown. Energies (in Kcal $\cdot \mathrm{mol}^{-1}$ ) 
Table 3. Kinetic parameters for steady-state reaction and for the pre-steady-state reductive half-readction of native AAO and three Tyr92 variants in the oxidation of 4-methoxybenzyl alcohol

\begin{tabular}{|c|c|c|c|c|c|c|c|}
\hline & \multicolumn{5}{|c|}{ Steady-state turnover } & \multicolumn{2}{|c|}{ Reductive half-reaction } \\
\hline & $\begin{array}{l}k_{\text {cat }} \\
\left(\mathrm{s}^{-1}\right)\end{array}$ & $\begin{array}{c}K_{\mathrm{m}(\mathrm{Al})} \\
(\mu \mathrm{M})\end{array}$ & $\begin{array}{l}k_{\mathrm{cat}} / K_{\mathrm{m}(\mathrm{Al})} \\
\left(\mathrm{s}^{-1} \mathrm{mM}^{-1}\right)\end{array}$ & $\begin{array}{c}K_{\mathrm{m}(\mathrm{Ox})} \\
(\mu \mathrm{M})\end{array}$ & $\begin{array}{l}k_{\text {caa }} / K_{\mathrm{m}(\mathrm{Ox})} \\
\left(\mathrm{s}^{-1} \mathrm{mM}^{-1}\right)\end{array}$ & $\begin{array}{l}k_{\text {red }} \\
\left(\mathrm{s}^{-1}\right)\end{array}$ & $\begin{array}{c}K_{\mathrm{d}} \\
(\mu \mathrm{M})\end{array}$ \\
\hline $\mathrm{AAO}^{1}$ & $129 \pm 5$ & $25 \pm 3$ & $5160 \pm 650$ & $348 \pm 36$ & $371 \pm 41$ & $115 \pm 3$ & $31 \pm 2$ \\
\hline Y92F & $120 \pm 1$ & $30 \pm 1$ & $4450 \pm 120$ & $147 \pm 4$ & $814 \pm 21$ & $119 \pm 9$ & $42 \pm 12$ \\
\hline Y92L & $100 \pm 2$ & $51 \pm 2$ & $1940 \pm 4$ & $348 \pm 13$ & $286 \pm 12$ & $95 \pm 2$ & $52 \pm 4$ \\
\hline Y92W & $11 \pm 0.3$ & $1890 \pm 70$ & $6 \pm 0.3$ & $181 \pm 7$ & $60 \pm 3$ & $14 \pm 1$ & $4630 \pm 520$ \\
\hline
\end{tabular}

Measurements were carried out in $0.1 \mathrm{M}$ phosphate buffer, $\mathrm{pH} 6$, at $12{ }^{\circ} \mathrm{C}$. Bisubstrate steady-state constants were determined by varying the concentrations of both alcohol and $\mathrm{O}_{2}$, and fitting the data to Eq. 1 (native AAO) or Eq. 2 (Y92F, Y92L and Y92W variants) describing ternary and ping-pong mechanisms, respectively (see Materials and Methods). Means and standard deviations are provided. ${ }^{1}$ Data from Ferreira et al. [14] 
Table 4. KIE on apparent steady-state parameters for the oxidation of three $\alpha$-deuterated $p$-methoxybenzyl alcohols by native AAO and its $\mathrm{Y} 92 \mathrm{~L}$ variant estimated under $\mathrm{O}_{2}-$ saturation conditions

\begin{tabular}{|c|c|c|c|}
\hline & ${ }^{\mathrm{D}}\left({ }^{a p p} k_{\mathrm{cat}}\right)$ & ${ }^{\mathrm{D}}\left({ }^{a p p} K_{\mathrm{m}(\mathrm{Al})}\right)$ & ${ }^{\mathrm{D}}\left({ }^{a p p} k_{\mathrm{cat}} / K_{\mathrm{m}(\mathrm{Al})}\right)$ \\
\hline \multicolumn{4}{|l|}{ Native $A A O^{I}$} \\
\hline$(R)-\left[\alpha-{ }^{2} \mathrm{H}\right]-4-$ Methoxybenzyl alcohol & $5.0 \pm 0.0$ & $1.3 \pm 0.1$ & $3.8 \pm 0.1$ \\
\hline (S)- $\left[\alpha-{ }^{2} \mathrm{H}\right]-4-$ Methoxybenzyl alcohol & $1.3 \pm 0.0$ & $1.0 \pm 0.1$ & $1.3 \pm 0.1$ \\
\hline$\left[\alpha-{ }^{2} \mathrm{H}_{2}\right]-4-M e t h o x y b e n z y l$ alcohol & $7.6 \pm 0.1$ & $1.9 \pm 0.1$ & $4.1 \pm 0.1$ \\
\hline Y92L variant: & & & \\
\hline$(R)-\left[\alpha-{ }^{2} \mathrm{H}\right]-4-M e t h o x y b e n z y l$ alcohol & $4.0 \pm 0.0$ & $1.0 \pm 0.1$ & 4.3. \pm 0.1 \\
\hline (S)- $\left[\alpha-{ }^{2} \mathrm{H}\right]-4-$ Methoxybenzyl alcohol & $1.3 \pm 0.0$ & $1.1 \pm 0.1$ & $1.2 \pm 0.1$ \\
\hline$\left[\alpha-{ }^{2} \mathrm{H}_{2}\right]-4-$ Methoxybenzyl alcohol & $6.2 \pm 0.1$ & $1.1 \pm 0.1$ & $5.8 \pm 0.1$ \\
\hline
\end{tabular}

Steady-state constants were estimated in $\mathrm{O}_{2}$-saturated $\left(1.279 \mathrm{mM} \mathrm{O}_{2}\right) 0.1 \mathrm{M}$ phosphate, $\mathrm{pH} 6$, at $25^{\circ} \mathrm{C}$. KIE values are the ratio between the activity on protiated and deuterated substrate calculated by fitting constants to Eq. 6 . Means and standard deviations are provided. ${ }^{1}$ Data from Ferreira et al. and Hernandez-Ortega et al. [14, 33] 
Scheme 1. Ternary and ping-pong mechanisms in AAO catalysis. Ternary and ping-pong mechanisms are described by the upper and lower loops, respectively, while the right reaction corresponds to aldehyde release in anaerobic experiments.

Figure 1. Detail of the AAO active site after alcohol substrate migration. Position of 4methoxybenzyl alcohol after migration to the AAO active site using the PELE software [52] (on 3FIM crystal structure). The alcohol situates in the environment of two conserved histidines, three aromatic residues, and the FAD isoalloxazine ring.

Figure 2. Bisubstrate steady-state kinetics for AAO. Double reciprocal plot for the oxidation of 3-chloro-4-methoxybenzyl (A) and 3-chlorobenzyl (B) alcohols by native AAO, measured in $0.1 \mathrm{M}$ phosphate buffer, $\mathrm{pH} 6$, at $12{ }^{\circ} \mathrm{C}$, as a function of the alcohol substrate concentration at fixed $\mathrm{O}_{2}$ concentrations: $61 \mu \mathrm{M}(\mathbf{x}), 152 \mu \mathrm{M}(\mathbf{\Delta}), 319 \mu \mathrm{M}(\bigcirc), 668 \mu \mathrm{M}(\mathbf{O})$ and $1520 \mu \mathrm{M}(\square)$. Direct plots are included in the corresponding insets.

Figure 3. AAO redox state during turnover with five alcohol substrates. The enzyme $(\sim 16$ $\mu \mathrm{M})$ was mixed in the stopped-flow spectrophotometer with equal volumes of $0.1 \mathrm{M}$ phosphate buffer, $\mathrm{pH}$ 6, containing $2 \mathrm{mM} 3$-chlorobenzyl alcohol (trace 1), $5 \mathrm{mM} 3$ fluorobenzyl-alcohol (trace 2), $1 \mathrm{mM}$ 3-chloro-4-methoxybenzyl alcohol (trace 3), $2 \mathrm{mM} 4$ methoxybenzyl alcohol (trace 4), $4 \mathrm{mM} \mathrm{3,4-dimethoxybenzyl} \mathrm{alcohol} \mathrm{(trace} \mathrm{5).} \mathrm{The} \mathrm{time}$ course of the reaction $\left(25^{\circ} \mathrm{C}\right)$ was monitored at $462 \mathrm{~nm}$.

Figure 4. AAO spectral changes during turnover. The AAO spectral changes during turnover experiments described in Fig. 3 with $1 \mathrm{mM}$ 3-chloro-4-methoxybenzyl alcohol (A) and $3 \mathrm{mM}$ 3-chlorobenzyl alcohol (B), are here shown. The oxidized enzyme sprectrum before mixing is shown as a dashed line. Spectra after mixing are indicated at 0.003, 0.011, $0.08,0.15,0.3,0.45,0.60,0.75,1,2,10,20$ and $40 \mathrm{~s}$ in $\mathbf{B}$, and at 0.16, 2.7, 4.3, 5, 10, 20, 30, 40, 50 and $60 \mathrm{~s}$ in $\mathbf{A}$. The corresponding insets show the spectral species $(A, B$ and $C)$ obtained after global analysis of the reactions. Revisar si estan cambiados los tiempos entre A y B

Figure 5. Pre-steady-state kinetics for the reductive half-reaction of AAO. Spectral timecourse of the anaerobic reduction of native AAO $(7.5 \mu \mathrm{M})$ by 3-chloro-4-methoxybenzyl (A) and 3-chlorobenzyl (B) alcohols. The spectrum of the oxidized enzyme before mixing is shown as a dashed line. Spectra after mixing are shown at 4, 19, 39, 80, 330 and $500 \mathrm{~ms}$ in $\mathbf{A}$, and at 2, 39, 78, 129, 219, $500 \mathrm{~ms}$ in $\mathbf{B}$. The corresponding insets show the absorbance spectra for the two-three kinetically distinguishable spectroscopic species $(A, B$ and $C)$ obtained by global analysis of the spectral evolution. The second inset shows the evolution of these species along the reaction course.

Figure 6. Pre-steady-state kinetics for the reductive half-reaction of three Tyr92 AAO variants when using as substrate 4-methoxybenzyl alcohol. Spectral evolution of the anaerobic reduction of Y92F (A), Y92L (B), and Y92W (C) AAO variants $(9 \mu \mathrm{M})$ by 4methoxybenzyl alcohol. Spectra for the oxidized enzyme before mixing are shown by dashed lines. Spectra after mixing are shown at 0.004, 0.006, 0.009, 0.0115, 0.0141, 0.0192 and $0.032 \mathrm{~s}$ in $\mathbf{A}, 0.004,0.006,0.012,0.017,0.029,0.099,0.25 \mathrm{~s}$ in $\mathbf{B}$, and 0.004, 0.006, 0.03, $0.11,0.18,0.44,0.98 \mathrm{~s}$ in $\mathbf{C}$. The corresponding insets show the spectra for the kinetically distinguishable species $(A$ and $B$ ) obtained by global analysis of the spectral evolution. Dependences of observed rates on the concentrations of 4-methoxybenzyl alcohol by native 
AAO $(\mathbf{O})$, Y92F $(O)$, Y92L $(\Delta)$ and Y92W $(\triangle)$ AAO variants are shown in $\mathbf{D}$ and fitted to Eq. 5. Assays performed at $12{ }^{\circ} \mathrm{C}$.

Figure 7. Logo of sequence of 70 putative AAO sequences from different basidiomycetes in positions 83-97 of mature $\boldsymbol{P}$. eryngii AAO. The compared sequences were taken from the following genomes available at JGI (www.jgi.doe.gov); the numbers of AAO sequences are indicated in parentheses): B. adusta (11), Dichomitus squalens (9), Fomitiporia mediterranea (1), Fomitopsis pinicola (1), Ganoderma sp. (7), Gelatoporia subvermispora (4), Gloeophyllum trabeum (2), Laccaria bicolor (1), P. chrysosporium (3), Phlebia brevispora (3), Punctularia strigosozonata (6), Rhodonia placenta (2), Stereum hirsutum (15) and Trametes versicolor (3). Numbers under each stack indicate the position in the mature $P$. eryngii AAO sequence; the overall height of the stack reflects the sequence conservation at that position; and the height of each symbol in the same column indicates the relative frequency of each amino acid. 
